\title{
A Combined Experimental and Numerical Approach That Eliminates the Non-uniqueness Associated With the Johnson-cook Parameters Obtained Using Inverse Methods
}

\author{
Nishant Ojal ( $\nabla$ nojal@uncc.edu ) \\ University of North Carolina at Charlotte \\ Harish P. Cherukuri \\ Tony L. Schmitz \\ Kyle T. Devlugt \\ Adam W. Jaycox
}

\section{Research Article}

Keywords: Johnson-Cook constitutive model, Extended Oxley model, Adaptive Memory Programming for Global Optimization, Particle Swarm Optimization, Orthogonal Machining, Finite Element Analysis

Posted Date: August 23rd, 2021

DOl: https://doi.org/10.21203/rs.3.rs-799799/v1

License: (c) (1) This work is licensed under a Creative Commons Attribution 4.0 International License. Read Full License

Version of Record: A version of this preprint was published at The International Journal of Advanced Manufacturing Technology on February 22nd, 2022. See the published version at https://doi.org/10.1007/s00170-021-08640-9. 


\title{
A combined experimental and numerical approach that eliminates the non-uniqueness associated with the Johnson-Cook parameters obtained using inverse methods.
}

\author{
Nishant Ojal • Harish P. Cherukuri - Tony L. Schmitz • Kyle T. \\ Devlugt • Adam W. Jaycox
}

Received: date / Accepted: date

\begin{abstract}
Johnson-Cook constitutive model is a commonly used material model for machining simulations. The model includes five parameters that capture the initial yield stress, strain-hardening, strain-rate hardening, and thermal softening behavior of the material. These parameters are difficult to determine using experiments since the conditions observed during machining (such as high strain-rates of the order of $10^{5} / \mathrm{sec}-$ $10^{6} / \mathrm{sec}$ ) are challenging to recreate in the laboratory. To address this problem, several researchers have recently proposed inverse approaches where a combination of experiments and analytical models are used to predict the Johnson-Cook parameters. The errors between the measured cutting forces, chip thicknesses and temperatures and those predicted by analytical models are minimized and the parameters are determined.
\end{abstract}

In this work, it is shown that only two of the five Johnson-Cook parameters can be determined uniquely using inverse approaches. Two different algorithms, namely Adaptive Memory Programming for Global Optimization (AMPGO) and Particle Swarm Optimization (PSO), are used for this purpose. The extended Oxley's model is used as the analytical tool for optimization. For de-

N. Ojal, H.P. Cherukuri

The University of North Carolina at Charlotte, Charlotte, NC 28223-0001, USA

Tel.: +1-704-805-0091

Fax: +1-704-687-8345

E-mail:nojal@uncc.edu

T. L. Schmitz

The University of Tennessee, Knoxville, Knoxville, TN 379962210, USA

K. T. Devlugt, A. W. Jaycox

Lawrence Livermore National Laboratory, Livermore, CA 94550, USA termining a parameter's value, a large range for each parameter is provided as an input to the algorithms. The algorithms converge to several different sets of values for the five Johnson-Cook parameters when all the five parameters are considered as unknown in the optimization algorithm. All of these sets, however, yield the same chip shape and cutting forces in FEM simulations. Further analyses show that only the strain-rate and thermal softening parameters can be determined uniquely and the three parameters present in the strainhardening term of the Johnson-Cook model cannot be determined uniquely using the inverse method. A combined experimental and numerical approach is proposed to eliminate this determine all parameters uniquely.

Keywords Johnson-Cook constitutive model · Extended Oxley model · Adaptive Memory Programming for Global Optimization · Particle Swarm Optimization · Orthogonal Machining · Finite Element Analysis

\section{Introduction}

Modeling and simulation are important tools for understanding and optimizing machining processes. Analytical models such as the Oxley machining model [1] and finite element simulations [2-6] are used by researchers for this purpose. Both approaches require a constitutive model for describing the material behavior at the extreme thermomechanical conditions present during machining. The most commonly used material model in machining is the Johnson-Cook constitutive model [7].

Using five material-dependent parameters, the JohnsonCook model relates flow stress with strain, strain-rate and temperature of the material. Experimentally, these 
parameters are obtained by fitting the data of the quasistatic tests at various temperatures and dynamic tests at different strain-rates. Tensile tests and Split-Hopkinson Pressure Bar (SHPB) tests are two of the techniques typically used for this purpose [8]. However, the extreme conditions of machining [9] such as strain-rates of the order $10^{5} / \mathrm{s}$ to $10^{6} / \mathrm{s}$ and high temperatures are challenging to achieve in these experiments. To overcome this difficulty, various researchers have proposed inverse approaches to determine these parameters using the data from the actual machining experiments. However, these methods have been shown to have the drawback of producing non-unique solutions to the JohnsonCook parameter values [10-12].

Machining conditions, experimental observations and simulation results (using finite element analyses or analytical models) are the input to the inverse methods. The simulation results depend on the material model parameters. The goal is to determine these material model parameters by minimizing the objective function consisting of the difference between experimental observations and simulation results. This minimization is achieved by tuning the parameters of the material model using an optimization algorithms. The determined material model parameter set is used in the computational or analytical models and validated with experimental results. Although experiments such as uniaxial, isothermal compression testing of cylindrical specimens [13], laser peening [14], and cold wire drawing [15] have been used for validation purposes, the most widely used experimental technique is based on orthogonal machining.

In this work, the source of non-unique solutions to the inverse problem is identified and an approach to determine a unique set of Johnson-Cook parameters is presented. The extended Oxley's model is used as the analytical model for simulation. Two separate optimization algorithms, Adaptive Memory Programming for Global Optimization (AMPGO) and Particle Swarm Optimization (PSO), are used to minimize the objective function. The consideration of two separate algorithms eliminates any bias that may be present in one particular method.

\section{Literature review}

During machining, the workpiece material undergoes plastic deformation. The plastic behaviour of the material is modelled using the Johnson-Cook material model:

$\sigma_{e q}=\left(A+B \varepsilon_{p}^{n}\right)\left[1+C \ln \left(\frac{\dot{\varepsilon_{p}}}{\dot{\varepsilon}_{0}}\right)\right]\left[1-\left(\frac{T-T_{0}}{T_{m}-T_{0}}\right)^{m}\right]$.
Here, $\sigma_{e q}$ is flow stress, $\varepsilon_{p}$ is the equivalent plastic strain, $\dot{\varepsilon}_{p}$ is the plastic strain-rate, $\dot{\varepsilon}_{0}$ is a reference strain-rate, $T_{m}$ is the melting temperature, and $T_{0}$ is a reference temperature. $A, B, C, n$ and $m$ are the material dependant parameters. $A$ denotes the initial yield strength, $B$ and $n$ describes the strain hardening, $C$ captures the strain-rate sensitivity and $m$ the thermal sensitivity of the material.

The majority of the existing studies that use the inverse approach for determining the Johnson-Cook parameters can be divided into three categories. In the first category, all five parameters of the Johnson-Cook model are taken as optimizing parameters and through optimization algorithms, determination of unique parameter set is claimed. Agmell et al.[16] and Ning et al. [17] performed inverse analysis using a Kalman filter and machining experiments. The relationship between the Johnson-Cook parameters and machining output parameters was developed through multiple FEM simulations. Five discrete values within a range of \pm 30 $\%$ of each Johnson-Cook parameter of reference material were used for these simulations. A close agreement between the simulated FEM results and experimental observations was observed using the parameter set obtained through the inverse approach. Ozel et al. [18] used different variations of the PSO method, namely, PSO, PSO-c and CPSO method, to determine the Johnson-Cook parameters. A unique set of parameters were obtained for each method, but the values differ for each method. The parameter set obtained using CPSO showed the best correlation with the experimental results. Eisseler et al. [19] used design of experiment (DoE) for inverse parameter identification by comparing the cutting force of FEM simulations with 50 Johnson-Cook parameter sets and machining experiments. Two sets of all the five Johnson-Cook parameters were determined for steel SAE-4142 within a maximum difference of less than $3 \%$.

The second category recognizes the non-uniqueness of the parameters obtained by the inverse approach and proposes some suggestions to counter this nonuniqueness. Denkena et al. [11] used Oxley's machining model and Particle Swarm Optimization (PSO) method using the cutting and thrust forces in an orthogonal slot milling experiment to tune the Johnson-Cook parameters. The optimization was performed without using tensile test data (i.e., all five parameters varied) and with the use of tensile test data (i.e., $A, B$ and $n$ values were obtained from tensile test data and $C$ and $n$ optimized using PSO method). Wide variation was observed in the resulting set of parameters obtained by the two methods. Local minima in the solution domain were suggested to be the reason for this observation. 
Karkalos and Markopoulos [12] used the Fireworks optimization algorithm for the determination of all the five parameters of Johnson-Cook constitutive material model parameters. Their suggestion was to choose the bounds for optimization variables carefully to avoid premature convergence to a solution far from the optimal point. Pujana et al. [10] varied four parameters $(B, n$, $C$ and $m$ ) of the Johnson-Cook model for the inverse analysis using deterministic minimization techniques. The selection of initial values was found to influence the optimized set of parameters. The use of regularly distributed function evaluations was proposed in order to reach the absolute minimum.

The third category focuses on the non-uniqueness study and attempts to eliminate this by adding experimental results to determine a unique parameter set. Shrot et al. [20] studied the non-unique identification of Johnson-Cook parameters by matching the results of machining experiments and finite element models. The parameters $A, B$ and $n$ were varied in a defined interval. The parameter sets were selected based on the closeness of effective stress-strain plots using the standard Johnson-Cook parameters and test parameters. Similar chip shapes and cutting forces were obtained by the FEM simulation using these identified parameter sets. However, the non-uniqueness was attributed to the measurement error during experiments. The use of different cutting conditions was suggested to obtain a unique parameter set.

Storchak et al. [21] determined the Johnson-Cook parameters using a combination of compression test and machining experiments. The parameters $A, B, n$ and $m$ were obtained using the compression tests at various strain-rates and temperatures. Only the parameter $C$ was obtained using the Oxley theory and machining experiments.

These three different approaches coupled with multiple methods to determining unique values of JohnsonCook parameters via inverse methods show that the origin of non-uniqueness of the parameters (when inverse methods are used) is far from settled. In this paper, we hope to address this issue by considering two optimization algorithms.

\section{Methodology}

For the inverse approach using orthogonal machining, the observations of machining experiments, the results of machining simulations or an analytical model and a wide range of the Johnson-Cook parameters are used as input. An objective function comprising of the experimental observations and results of the simulation or analytical model is formed. The extended Oxley model is used in this work as the analytical model. This model predicts the machining output for a given set of the Johnson-Cook parameters and the machining conditions. Using the optimization algorithm, the Johnson-Cook parameters are tuned to minimize the objective function. The cutting force $\left(F_{c}\right)$, chip thickness ratio $\left(h_{c}\right)$ and tool-workpiece interface temperature $\left(T_{c}\right)$ are commonly taken as the variables of the objective function, which is given by

$$
\begin{aligned}
\operatorname{Err}=[ & \left(\frac{F_{c, \exp }-F_{c, s i m}}{F_{c, \exp }}\right)^{2}+\left(\frac{h_{c, \exp }-h_{c, s i m}}{h_{c, \exp }}\right)^{2} \\
& \left.+\left(\frac{T_{c, \exp }-T_{c, s i m}}{T_{c, \exp }}\right)^{2}\right]^{\frac{1}{2}}
\end{aligned}
$$

Here, the label "exp" represents experimental observation and "sim" represents simulated or calculated value. The observations of the machining experiment is assumed to be the same as that obtained from the extended Oxley model using the known values of JohnsonCook parameters in the literature [22]. This is done to eliminate the measurement uncertainty associated with experiments. Also, the accuracy of the determined Johnson-Cook parameters can be obtained by comparing with these reference values.

For investigating the reason behind multiple sets of Johnson-Cook parameters obtained using inverse approaches, two approaches (shown in Fig. 1) are taken. In the first approach, all the five Johnson-Cook parameters are considered as the unknown variables. In the second approach, the parameters $C$ and $m$ are considered as the only unknown variables. The motivation behind this is given in Section 5. The parameters $A, B$ and $n$ are considered as known. They are determined using the quasi-static tensile test and hence their values are fixed. For the unknown Johnson-Cook parameters $C$ and $m$, a wide range is provided as input to the optimization algorithm. This is based on their values for different materials in the literature [7] and [23]. The ranges considered for the parameters are shown in Table 1. The machining conditions used as input parameters are given in Table 2 .

The Johnson-Cook parameters are obtained with these inputs by minimizing the objective function using the optimization algorithms, AMPGO, and PSO algorithms. In the optimization loop, the extended Oxley model is used to calculate the values of the machining output for a given set of Johnson-Cook parameters. The output of Approach 1 is all the five parameters, whereas the parameters $C$ and $m$ are the output of Approach 2. The uniqueness of the parameter sets is discussed in the results section. 


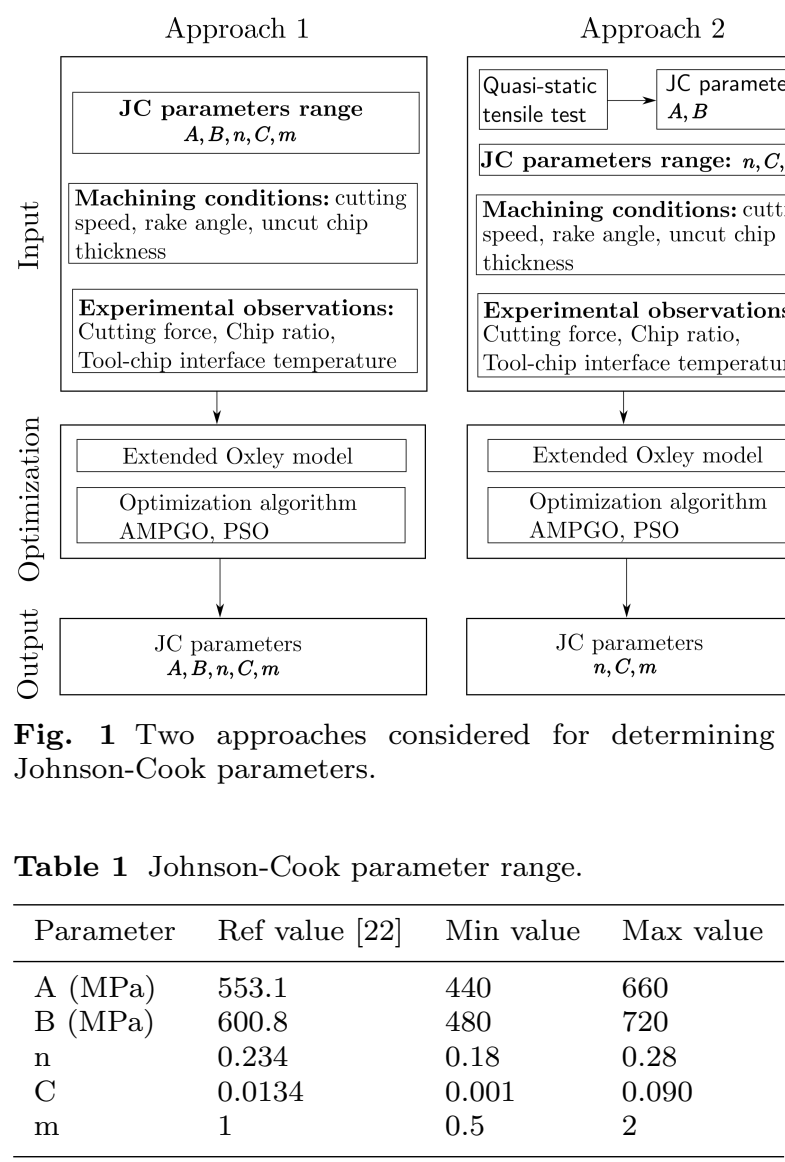

Table 2 Machining conditions used [24].

\begin{tabular}{lllr}
\hline Test no. & $V(\mathrm{~m} / \mathrm{min})$ & $t_{1}(\mathrm{~mm})$ & $\alpha\left(^{\circ}\right)$ \\
\hline 1 & 200 & 0.15 & -7 \\
2 & 200 & 0.15 & 5 \\
3 & 200 & 0.30 & -7 \\
4 & 200 & 0.30 & 5 \\
5 & 400 & 0.15 & -7 \\
6 & 400 & 0.15 & 5 \\
7 & 400 & 0.30 & -7 \\
8 & 400 & 0.30 & 5 \\
\hline
\end{tabular}

\subsection{Extended Oxley theory}

The parallel-sided shear zone theory, proposed by Oxley [1] is a widely used analytical model for predicting the stresses, cutting forces and the average temperature in orthogonal machining. The deformation zone consists of two regions: a parallel-sided primary shear zone and a rectangular secondary shear zone at the tool-chip interface of constant thickness. Along with the application of the principle of minimum work, this theory analyses the equilibrium of the shear plane $\mathrm{AB}$ (shown in Fig. 2) and the tool-chip interface to determine the physical parameters of the machining process. This is done by tuning three parameters, namely shear angle $\phi$, strain- rate constant at the shear zone $C_{0}$ and strain-rate constant at the tool-chip interface zone $\delta$.

\subsubsection{Shear Plane $A B$}

Let $t_{1}$ be the uncut chip thickness, $V$ the cutting velocity and $\alpha$ the rake angle of the tool. Then the length of the shear plane $l_{A B}$, the chip thickness $t_{2}$, the shear velocity $V_{S}$, and the chip velocity $V_{C}$ are

$$
\begin{aligned}
l_{A B} & =\frac{t_{1}}{\sin \phi}, \\
t_{2} & =\frac{t_{1} \cos (\phi-\alpha)}{\sin \phi}, \\
V_{S} & =\frac{V \cos \alpha}{\cos (\phi-\alpha)},
\end{aligned}
$$

$V_{C}=\frac{V \sin \phi}{\cos (\phi-\alpha)}$.

The equivalent shear strain at $\mathrm{AB}, \varepsilon_{A B}$, is

$\varepsilon_{A B}=\frac{\gamma_{A B}}{\sqrt{3}}=\frac{\cos \alpha}{2 \sqrt{3} \sin \phi \cos (\phi-\alpha)}$.

The equivalent shear strain-rate along $\mathrm{AB}, \dot{\varepsilon}_{A B}$, is

$\dot{\varepsilon}_{A B}=\frac{\dot{\gamma}_{A B}}{\sqrt{3}}=\frac{C_{0} V_{S}}{\sqrt{3} l_{A B}}$.

The shear flow stress $k_{A B}$ can be obtained from the tensile flow stress $\sigma_{A B}$ by using the von Mises criterion and the Johnson-Cook material model as

$k_{A B}=\frac{\sigma_{A B}}{\sqrt{3}}$

with

$\sigma_{A B}=\left(A+B \varepsilon_{A B}^{n}\right)\left[1+C \ln \left(\frac{\dot{\varepsilon}_{A B}}{\dot{\varepsilon}_{0}}\right)\right]\left[1-\left(\frac{T_{A B}-T_{0}}{T_{m}-T_{0}}\right)^{m}\right]$.

The average temperature along $\mathrm{AB}, T_{A B}$ is calculated using

$T_{A B}=T_{0}+\eta \Delta T_{S Z}$

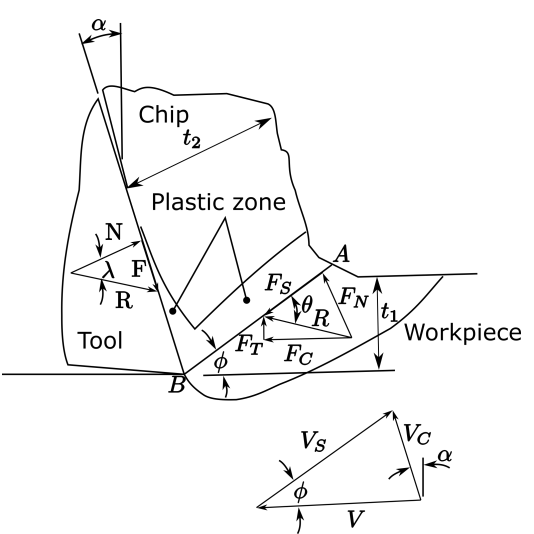

Fig. 2 Chip formation model in Oxley's theory [1]. 
Here, $T_{0}$ is the initial temperature, $\eta$ is the Taylor-Quinney coefficient and $\Delta T_{S Z}$ is the temperature rise in the primary shear zone. This temperature rise is obtained using the plastic work done in shear zone and is given by

$\Delta T_{S Z}=\frac{(1-\beta) F_{S} V_{S}}{\rho V t_{1} w C_{p}}$.

Here, $\beta$ is the fraction of heat conducted into the workpiece from the shear zone, which is estimated from the empirical equations

$\beta=0.5-0.35 \log \left(R_{T} \tan \phi\right)$ for $0.04 \leq R_{T} \tan \phi \leq 10$ $=0.3-0.15 \log \left(R_{T} \tan \phi\right)$ for $R_{T} \tan \phi \geq 10$.

Here $R_{T}$ is a non-dimensional number given in terms of the specific heat $\left(C_{p}\right)$, cutting velocity $(V)$, uncut chip thickness $\left(t_{1}\right)$ and the thermal conductivity of the workpiece $(K)$ by

$R_{T}=\frac{\rho C_{p} V t_{1}}{K}$

The angles in the chip formulation model (Fig. 2) are related by the expressions

$\theta=\tan ^{-1}\left(1+2\left(\frac{\pi}{4}-\phi\right)-C_{0} n_{e q}\right)$,

and

$\lambda=\theta-\phi+\alpha$

Here, the strain hardening exponent for the JohnsonCool material model [24], $n_{e q}$ is expressed by the expression

$n_{e q} \approx \frac{n B \varepsilon_{A B}^{n}}{A+B \varepsilon_{A B}^{n}}$

The normal stress at the tool-chip interface is given by

$\sigma_{N}^{\prime}=k_{A B}\left(1+\frac{\pi}{2}-2 \alpha-2 C_{0} n_{e q}\right)$.

\subsubsection{Tool-chip interface}

The strain $\left(\varepsilon_{\text {int }}\right)$ and strain-rate $\left(\dot{\varepsilon}_{\text {int }}\right)$ at tool-chip interface using von Mises criterion are given by

$\varepsilon_{i n t}=\frac{\gamma_{i n t}}{\sqrt{3}}=2 \varepsilon_{A B}+\frac{h}{2 \sqrt{3} \delta t_{2}}$

and

$\dot{\varepsilon}_{i n t}=\frac{\gamma_{i n t}}{\sqrt{3}}=\frac{V_{C}}{\sqrt{3} \delta t_{2}}$.
The average temperature at the tool-chip interface, $T_{\text {int }}$ is given by

$T_{i n t}=T_{0}+\Delta T_{S Z}+\psi \Delta T_{M}$

Here, $\Delta T_{M}$ is the maximum temperature rise of the chip and is calculated using the empirical equation proposed by Boothroyd [25]:

$\log \left(\frac{\Delta T_{M}}{\Delta T_{C}}\right)=0.06-0.195 \delta \sqrt{\frac{R_{T} t_{2}}{t_{1}}}+0.5 \log \left(\frac{R_{T} t_{2}}{h}\right)$

The tool-chip interface length, $h$ and the average temperature rise in the chip $\Delta T_{C}$ are given by

$h=\frac{t_{1} \sin \theta}{\cos \lambda \sin \phi}\left(1+\frac{C_{0} n_{e q}}{3\left(1+2\left(\frac{\pi}{4}-\phi\right) C_{0} n_{e q}\right)}\right)$

and

$\Delta T_{C}=\frac{F V_{C}}{\rho V t_{1} w C_{p}}$

The flow stress at the tool-chip interface, $k_{c h i p}$ is calculated using the expression

$$
\begin{aligned}
k_{\text {chip }}=\frac{1}{\sqrt{3}}\left(A+B \varepsilon_{i n t}^{n}\right) & {\left[1+C \ln \left(\frac{\dot{\varepsilon}_{i n t}}{\dot{\varepsilon}_{0}}\right)\right] } \\
\times & {\left[1-\left(\frac{T_{i n t}-T_{0}}{T_{m}-T_{0}}\right)^{m}\right] . }
\end{aligned}
$$

\subsubsection{Cutting forces}

The cutting forces (shown in Fig. 2) are obtained using the equations

$$
\begin{aligned}
F & =R \sin \lambda, \\
N & =R \cos \lambda, \\
F_{S} & =R \cos (\phi+\lambda-\alpha)=k_{A B} l_{A B} w, \\
N_{S} & =R \sin (\phi+\lambda-\alpha), \\
F_{c} & =R \cos (\lambda-\alpha), \\
F_{t} & =R \sin (\lambda-\alpha) .
\end{aligned}
$$

The normal stress $\sigma_{n}$ and the shear stress $\tau_{\text {int }}$ at the tool-chip interface are given by

$\sigma_{N}=\frac{N}{h w}$

and

$\tau_{\text {int }}=\frac{F}{h w}$. 


\subsection{Calculation flowchart}

The flowchart for determining the Johnson-Cook parameters is shown in Fig. 3. There are two loops in the flowchart: the inner loop shown by the dashed line and the outer loop shown by the solid line. The calculation for the machining parameters $C_{0}, \phi$, and $\delta$ for a given Johnson-Cook parameter set is done in the inner loop. The objective of the inner loop is to minimizing the gap between $k_{\text {chip }}$ and $\tau_{\text {int }}, \sigma_{N}$ and $\sigma_{N}^{\prime}$ and the minimum cutting force $F_{C}$ by tuning the parameters $\delta, C_{0}$ and $\phi$. The detailed derivations and the equations used have been adopted from Lalwani et al. [24]. The difference in the calculation approach in the current work with respect to Lalwani et al. [24] is that instead of varying the values of the parameters $\left(\delta, C_{0}\right.$ and $\left.\phi\right)$ by discrete values, the optimization is done using the AMPGO algorithm. This eliminates the dependence of the optimization accuracy on the discrete interval.

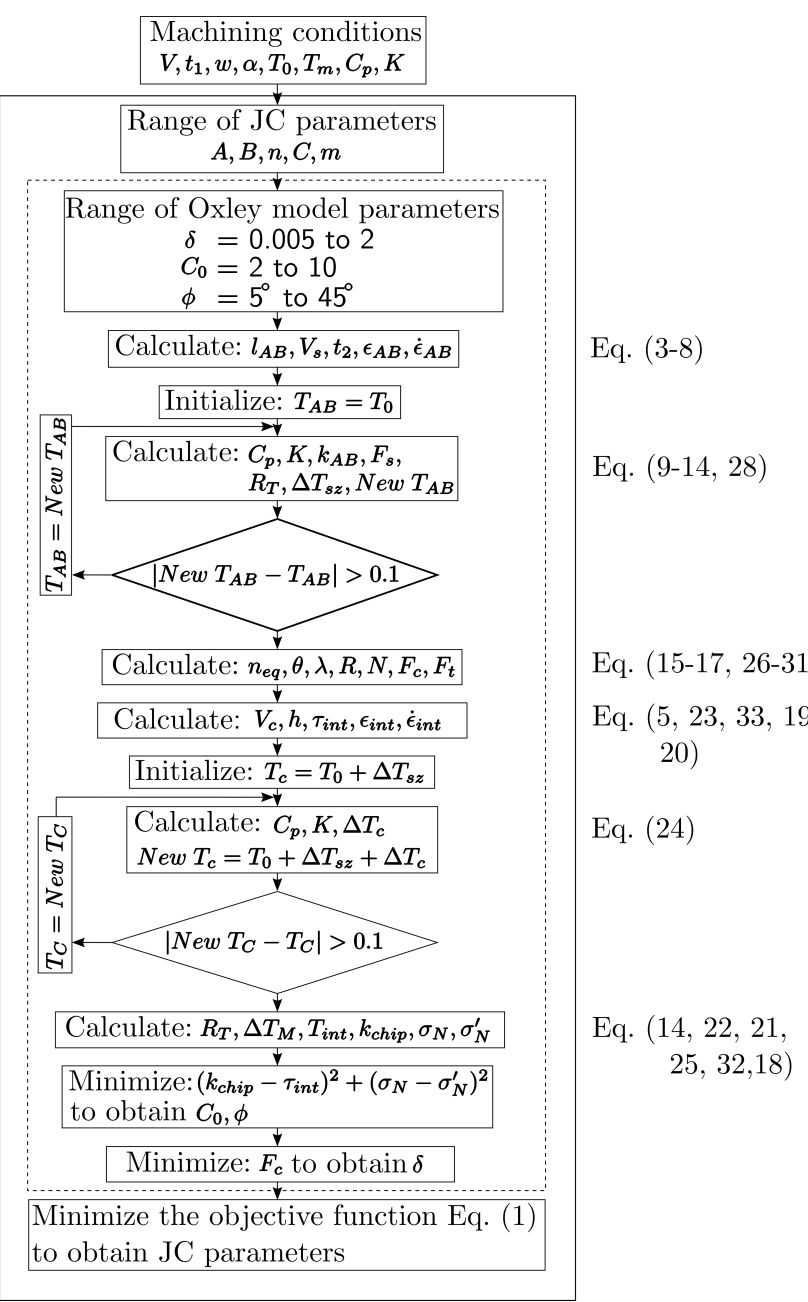

Fig. 3 Determining JC parameters using AMPGO/PSO method.

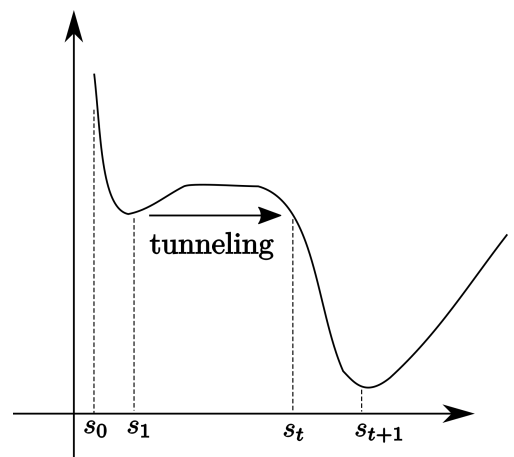

Fig. 4 Tunneling approach of AMPGO algorithm [26].

The optimal Johnson-Cook parameter values are determined using the outer loop. A wide range of JohnsonCook parameters is provided as input. The objective here is to minimize the objective function of Eq. (2) by tuning the Johnson-Cook parameters. The minimization is accomplished using both the AMPGO algorithm and PSO algorithm for each of the two approaches shown in Fig. 1.

\subsection{Adaptive Memory Programming for Global Optimization (AMPGO) method}

Adaptive Memory Programming for Global Optimization (AMPGO) is an optimization algorithm presented by Lasdon et al. [26] for the constrained global optimization problems. It consists of two phases: minimization and tunneling. A local minimum is found using a descent algorithm. Then, using a tunneling loop, the objective is to improve the found minimum with the new solution away from the current solution for the next minimization phase. The tunneling function is given by the Eq. (34).

$\operatorname{TTf}(\mathbf{x})=\frac{(f(\mathbf{x})-\text { aspiration })^{2}}{\prod_{\mathbf{s}_{i} \in \text { Tabulist }}\left(\operatorname{dist}\left(\mathbf{x}, \mathbf{s}_{i}\right)\right)^{2}}$.

Here, $f(\mathbf{x})$ is the function to be optimized. The aspiration value for the objective function is slightly less than the current best value. Tabulist contains the points from which to move away, i.e., the most recent local solution and recent solutions of tunneling sub-problems that failed to achieve the optimum condition. Also, $\operatorname{dist}\left(\mathbf{x}, \mathbf{s}_{i}\right)$ denotes the Eulerian distance.

\subsection{Particle Swarm Optimization (PSO) method}

The particle swarm optimization method was first proposed by Kennedy and Eberhart [27]. The method, motivated by the social behavior of bird flocks, can be used 


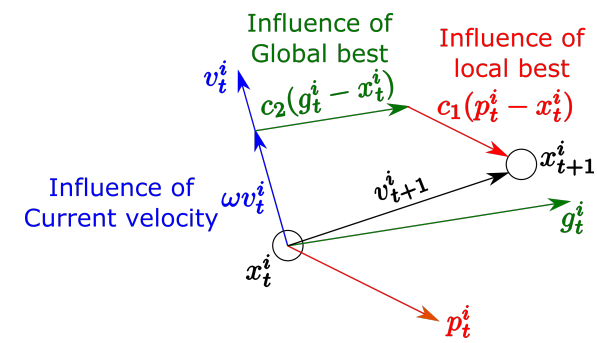

Fig. 5 Movement of particles in PSO algorithm.

to find the minimum or maximum values of an objective function $f(\mathbf{X})$, where $\mathbf{X}=\left[x_{1}, x_{2}, \ldots, x_{n}\right]$ is a vector of variables, also known as a position vector. In this method, a swarm size is chosen. The optimized solution is found based on the cooperation of these swarm particles in terms of learning and communication among them.

The search movement for optimum solution of each particle for an iteration (shown in Fig. 5 comprises of a component of current velocity direction $v_{t}^{i}$, a movement component towards local or personal best solution from all the previous iterations $c_{1}\left(p_{t}^{i}-x_{t}^{i}\right)$ and a movement component towards global best, i.e. the best solution of all the particles in the swarm $c_{2}\left(g_{t}^{i}-x_{t}^{i}\right)$. The velocity for the next iteration $(t+1)$ for $i^{t h}$ particle of the swarm, represented by $v_{t+1}^{i}$ is given by Eq. (35).

$v_{t+1}^{i}=\omega v_{t}^{i}+c_{1} r_{1}\left(p_{t}^{i}-x_{t}^{i}\right)+c_{2} r_{2}\left(g_{t}^{i}-x_{t}^{i}\right)$.

Here, parameter $\omega$ is called inertia weight, $c_{1}$ is called cognitive learning factor and $c_{2}$ is called social learning factor. These three parameters control the contribution of each factor in a particle's movement. The numbers $r_{1}$ and $r_{2}$ are the random numbers in the range $(0,1)$ and are used to avoid premature convergence. For this work, a swarm size of 20 with equal values to the parameters $c_{1}$ and $c_{2}$ is used.

\section{Results}

Using the first approach, multiple sets of optimal JohnsonCook parameters are obtained by both of the optimization algorithms. As an example, ten sets of optimal parameters for the machining condition 3 of Table 2 by AMPGO algorithm are given in Table 3 . The variation of each parameter over the ten sets is quite large. For example, the parameter $A$ varies from $449.5 \mathrm{MPa}$ in set 1 to $658.3 \mathrm{MPa}$ in set 10 .

Using the second approach, where only $C$ and $m$ are varied, very close values of the Johnson-Cook parameters are obtained by each of the algorithms. The optimization is done ten times for each of the machining conditions to verify the uniqueness of the parameter set
Table 3 Johnson-Cook parameters obtained using Approach 1 by AMPGO method (for Machining condition 3 ).

\begin{tabular}{lllll}
\hline$A(\mathrm{MPa})$ & $B(\mathrm{MPa})$ & $n$ & $C$ & $m$ \\
\hline 449.5 & 720.3 & 0.228 & 0.0160 & 0.922 \\
471.8 & 720.8 & 0.233 & 0.0140 & 0.921 \\
473.9 & 720.8 & 0.231 & 0.0130 & 0.935 \\
479.4 & 719.9 & 0.234 & 0.0130 & 0.924 \\
499.1 & 719.9 & 0.238 & 0.0110 & 0.929 \\
508.9 & 719.6 & 0.241 & 0.0100 & 0.924 \\
545.1 & 648.3 & 0.248 & 0.0120 & 0.951 \\
567.8 & 623.5 & 0.254 & 0.0110 & 0.959 \\
578.9 & 613.4 & 0.254 & 0.0100 & 0.978 \\
658.3 & 507.6 & 0.277 & 0.0100 & 1.024 \\
\hline
\end{tabular}

Table 4 JC parameters obtained using Approach 2 by AMPGO.

\begin{tabular}{lll}
\hline Test no. & $\mathrm{C}$ & $\mathrm{m}$ \\
\hline 1 & 0.01343 & 0.9987 \\
2 & 0.01342 & 0.9990 \\
3 & 0.01345 & 0.9988 \\
4 & 0.01347 & 0.9983 \\
5 & 0.01337 & 0.9999 \\
6 & 0.01346 & 0.9979 \\
7 & 0.01349 & 0.9982 \\
8 & 0.01353 & 0.9970 \\
Jaspers and Dautzenberg [22] & 0.0134 & 1 \\
\hline
\end{tabular}

Table 5 JC parameters obtained using Approach 2 by PSO.

\begin{tabular}{lll}
\hline Test no. & $\mathrm{C}$ & $\mathrm{m}$ \\
\hline 1 & 0.01344 & 0.9986 \\
2 & 0.01342 & 0.9990 \\
3 & 0.01346 & 0.9986 \\
4 & 0.01347 & 0.9982 \\
5 & 0.01339 & 0.9997 \\
6 & 0.01348 & 0.9975 \\
7 & 0.01356 & 0.9970 \\
8 & 0.01354 & 0.9969 \\
Jaspers and Dautzenberg [22] & 0.0134 & 1 \\
\hline
\end{tabular}

obtained by each algorithm. The deviation of the obtained parameters is within $\pm 2 \%$ from the values of the parameters in the literature [22]. The average value of ten optimizations for each of the parameters obtained by the AMPGO and PSO algorithm is given in Table 4 and Table 5 respectively.

Comparisons of the optimal values of the Johnson Cook parameters $C$ and $m$ from the optimization algorithms AMPGO and PSO are shown in Figs. 6 and 7 respectively. The solid red lines in the plots represent the range of the parameter provided as an input to the algorithm. Also, the green dashed line indicates the value reported in the literature [22]. The results indicate that the optimization algorithms match the value provided in the literature to a fair accuracy. 


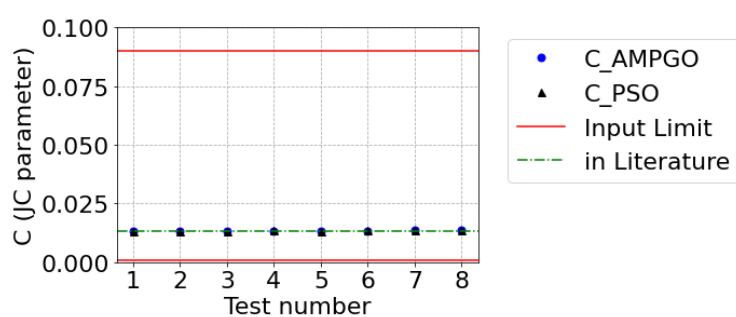

Fig. 6 Comparison of Johnson Cook parameter $C$.

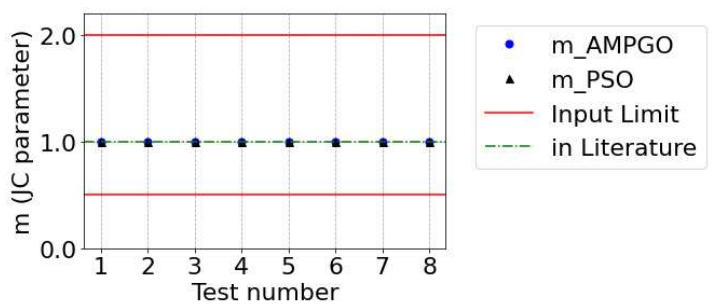

Fig. 7 Comparison of Johnson Cook parameter $m$.

Table 6 Strain hardening term for Johnson-Cook parameters obtained using Approach 1 for the machining condition 3.

\begin{tabular}{lllll}
\hline$A(\mathrm{MPa})$ & $B(\mathrm{Mpa})$ & $n$ & $\varepsilon_{p}$ & $A+B \varepsilon_{p}^{n}$ \\
\hline 449.508 & 720.342 & 0.2276 & 0.6321 & 1098 \\
471.753 & 720.800 & 0.2328 & 0.6321 & 1120 \\
473.947 & 720.798 & 0.2306 & 0.6320 & 1122 \\
479.400 & 719.891 & 0.2341 & 0.6321 & 1126 \\
499.116 & 719.877 & 0.2376 & 0.6321 & 1145 \\
508.910 & 719.630 & 0.2408 & 0.6321 & 1153 \\
545.087 & 648.332 & 0.2482 & 0.6321 & 1124 \\
567.799 & 623.450 & 0.2541 & 0.6321 & 1123 \\
578.884 & 613.379 & 0.2539 & 0.6320 & 1125 \\
658.250 & 507.556 & 0.2775 & 0.6321 & 1105 \\
\hline
\end{tabular}

\section{Discussion on the unique determination of Johnson-Cook parameters}

As discussed in the Results section, non-unique sets of the Johnson-Cook parameters are obtained when all the five Johnson-Cook parameters are chosen as unknowns. To investigate further, the machining quantities calculated using these Johnson-Cook parameters sets are compared in Table 7 . The variables of the objective function, i.e., the cutting force, chip ratio and the tool-chip interface temperature, agree for all ten sets. In fact, the values of all the other quantities such as the shear strain $\varepsilon_{A B}$ and strain-rate $\dot{\varepsilon}_{A B}$ and average temperature $T_{A B}$ at shear plane $\mathrm{AB}$ match. This clearly shows that Approach 1 leads to multiple sets of values for the Johnson-Cook parameters while providing the same output.

To further confirm the non-uniqueness resulting from Approach 1, finite element simulations are carried out for Test case 2 using different parameter sets. The pre- dicted cutting forces from these simulations are compared in Fig. 8 and the corresponding chip profiles are shown in Fig. 9. Clearly, the results obtained from the simulations using different Johnson-Cook parameter sets are in close agreement.

To investigate the reason for the non-uniqueness of the Johnson-Cook parameters determined using Approach 1, the strain hardening term of the JohnsonCook constitutive model, i.e., $\left(A+B \varepsilon_{p}^{n}\right)$ is calculated for the ten parameters sets in Table 3 . It may be recalled that these parameter sets were obtained using Approach 1 for the machining condition 3 of Table 2. The calculated strain hardening term for each of these sets is shown in Table 6. It is observed that in all the cases, this term has approximately the same value. Thus, it may be concluded that the inverse methods discussed here determine the strain-hardening term uniquely but are not able to determine the parameters $A, B$, and $n$ uniquely.

A unique set of Johnson-Cook parameters is obtained using Approach 2 where the parameters $A, B$ and $n$ are determined using the tensile test experiment and the parameters $C$ and $m$ are determined using the inverse method. Thus to determine the Johnson-Cook parameters uniquely, an inverse method comprising of the quasi-static tensile test, orthogonal machining experiments, analytical models or simulations and optimization algorithms can be followed.

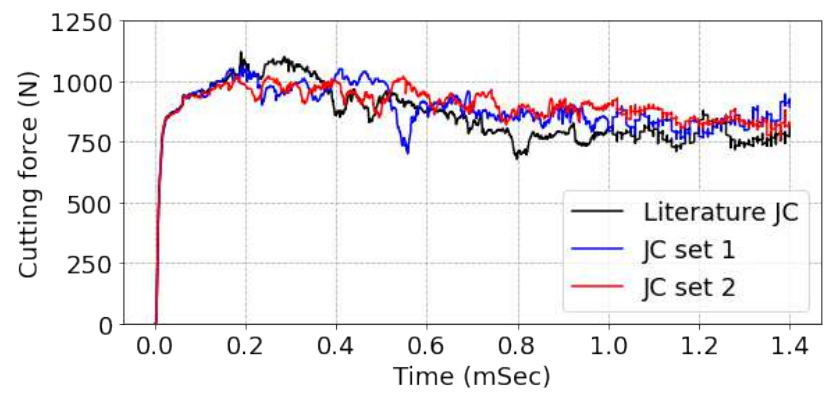

Fig. 8 Comparison of cutting force using different JC parameter sets.

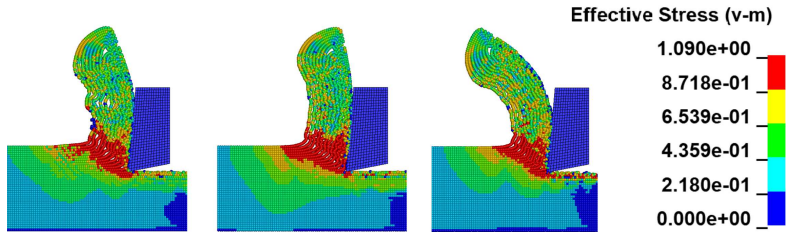

Fig. 9 Comparison of chip profile using different JC parameter sets. 
Table 7 Machining quantities calculated using Johnson-Cook parameters using Approach 1.

\begin{tabular}{|c|c|c|c|c|c|c|c|c|c|c|c|c|c|c|}
\hline $\begin{array}{l}t_{2} \\
(\mathrm{~mm})\end{array}$ & $\varepsilon_{A B}$ & $\begin{array}{l}\varepsilon_{\dot{A} B} \\
(1 / \mathrm{s})\end{array}$ & $\varepsilon_{\text {int }}$ & $\begin{array}{l}\varepsilon_{\text {int }} \\
(1 / \mathrm{s})\end{array}$ & $\begin{array}{l}T_{A B} \\
\left({ }^{\circ}\right. \\
\mathrm{C})\end{array}$ & $\begin{array}{l}T_{i n t} \\
\left(\begin{array}{c}\circ \\
\end{array}\right)\end{array}$ & $\begin{array}{l}k_{A B} \\
\left(N / m m^{2}\right)\end{array}$ & $n_{e q}$ & $\theta^{\circ}$ & $\begin{array}{l}\sigma_{N} \\
\left(N / m m^{2}\right)\end{array}$ & $\begin{array}{l}h \\
(\mathrm{~mm})\end{array}$ & $\begin{array}{l}F_{c} \\
(\mathrm{~N})\end{array}$ & $\begin{array}{l}F_{t} \\
(\mathrm{~N})\end{array}$ & $\begin{array}{l}\text { Chip } \\
\text { ratio }\end{array}$ \\
\hline 0.54 & 0.63 & 11583 & 14.72 & 92995 & 292.4 & 1015.0 & 573.8 & 0.13 & 47.16 & 871.5 & 0.5 & 770.7 & 235.3 & 1.8 \\
\hline 0.54 & 0.63 & 11566 & 14.68 & 92733 & 292.5 & 1014.8 & 573.9 & 0.13 & 47.16 & 871.6 & 0.5 & 770.7 & 235.3 & 1.8 \\
\hline 0.54 & 0.63 & 11692 & 14.87 & 94064 & 292.4 & 1015.4 & 573.8 & 0.13 & 47.16 & 871.5 & 0.5 & 770.5 & 235.2 & 1.8 \\
\hline 0.54 & 0.63 & 11590 & 14.70 & 92869 & 292.4 & 1014.9 & 573.9 & 0.13 & 47.16 & 871.6 & 0.5 & 770.7 & 235.3 & 1.8 \\
\hline 0.54 & 0.63 & 11625 & 14.74 & 93150 & 292.4 & 1015.0 & 573.8 & 0.13 & 47.16 & 871.6 & 0.5 & 770.6 & 235.3 & 1.8 \\
\hline 0.54 & 0.63 & 11578 & 14.66 & 92556 & 292.5 & 1014.7 & 573.9 & 0.13 & 47.16 & 871.6 & 0.5 & 770.8 & 235.4 & 1.8 \\
\hline 0.54 & 0.63 & 12189 & 14.66 & 92549 & 292.5 & 1014.7 & 573.9 & 0.13 & 47.16 & 871.6 & 0.5 & 770.8 & 235.4 & 1.8 \\
\hline 0.54 & 0.63 & 12401 & 14.60 & 92174 & 292.5 & 1014.5 & 573.9 & 0.13 & 47.16 & 871.6 & 0.5 & 770.8 & 235.4 & 1.8 \\
\hline 0.54 & 0.63 & 12641 & 14.79 & 93519 & 292.4 & 1015.2 & 573.8 & 0.12 & 47.16 & 871.5 & 0.5 & 770.6 & 235.2 & 1.8 \\
\hline 0.54 & 0.63 & 13882 & 14.68 & 92716 & 292.4 & 1014.8 & 573.9 & 0.11 & 47.16 & 871.6 & 0.5 & 770.7 & 235.3 & 1.8 \\
\hline
\end{tabular}

\section{Conclusion}

For simulating the behavior of materials during various manufacturing operations such as machining, the Johnson-Cook material model is widely used. Due to the limitation in achieving the extreme conditions of the actual operation, the parameters of the material model determined using experiments may not be accurate. Various researchers use the inverse method to overcome this difficulty. This work evaluates the uniqueness of the parameters obtained from the inverse method. Nonunique parameter sets are obtained using the current approach used by the researchers. The reason for the non-unique determination is identified. A combined experimental and numerical approach is presented to determine the parameters of the Johnson-Cook constitutive model uniquely. This approach uses a combination of analytical models, experiments, and optimization algorithms. The following conclusions can be made:

1. When all five parameters of the Johnson-Cook model are selected as unknowns, multiple sets of values are predicted by the inverse approach.

2. The proposed solution to the lack of uniqueness of solutions in the inverse approach is to determine the parameters $A, B$ and $n$ from quasi-static tensile tests and to determine $C$ and $m$ using the inverse approach.

3. A benefit of this approach is that orthogonal machining experiments along with uniaxial, quasistatic tensile tests are sufficient for determining the JohnsonCook parameters. The difficulty of conducting experiments at the extreme high strain-rates and high temperatures observed in machining to determine the thermal softening and strain-rate parameters $C$ and $m$ is eliminated.

4. The proposed inverse approach converges to the right values even when the search space for each of the parameters, $C$ and $m$, is large.

\section{Declarations}

Funding This work was performed under the auspices of the U.S. Department of Energy by Lawrence Livermore National Laboratory under Contract DE-AC52$07 \mathrm{NA} 27344$.

Conflicts of interest The authors declare no competing interests.

Availability of data and material All data generated and analyzed during the study are included in this article.

Authors' contributions Nishant Ojal conducted this study, analyzed the data and drafted the manuscript. Dr. Cherukuri supervised this work. Dr. Schmidt contributed to the literature review. Kyle and Adam provided critical feedback and helped shape the research. All authors have read and agreed to the published version of the manuscript.

\section{Ethics approval Not applicable}

\section{Consent to participate Not applicable}

Consent for publication Not applicable

\section{References}

1. Peter Louis Brennan Oxley and Milton C Shaw. Mechanics of Machining: An Analytical Approach to Assessing Machinability. Ellis Horwood Ltd.,Chichester, U.K. division of John Wiley and Sons, 1990.

2. B Zhang and A Bagchi. Finite element simulation of chip formation and comparison with machining experiment. Journal of Manufacturing Science and Engineering, 1994. 
3. John T Carroll III and John S Strenkowski. Finite element models of orthogonal cutting with application to single point diamond turning. International Journal of Mechanical Sciences, 30(12):899920, 1988.

4. Pedro J Arrazola et al. Investigations on the effects of friction modeling in finite element simulation of machining. International Journal of $\mathrm{Me}$ chanical Sciences, 52(1):31-42, 2010.

5. M Movahhedy, MS Gadala, and Y Altintas. Simulation of the orthogonal metal cutting process using an arbitrary lagrangian-eulerian finite-element method. Journal of Materials Processing Technology, 103(2):267-275, 2000.

6. Tugrul Özel. The influence of friction models on finite element simulations of machining. International Journal of Machine Tools and Manufacture, 46(5):518-530, 2006.

7. Gordon R Johnson and William H Cook. A constitutive model and data for metals subjected to large strains, high strain rates and high temperatures. In Proceedings of the 7th International Symposium on Ballistics, volume 21, pages 541-547. The Netherlands, 1983.

8. Herbert Kolsky. An investigation of the mechanical properties of materials at very high rates of loading. Proceedings of the Physical Society. Section B, 62(11):676, 1949.

9. PJ Arrazola, T Özel, D Umbrello, M Davies, and IS Jawahir. Recent advances in modelling of metal machining processes. CIRP Annals, 62(2):695-718, 2013.

10. J Pujana, PJ Arrazola, R M'saoubi, and H Chandrasekaran. Analysis of the inverse identification of constitutive equations applied in orthogonal cutting process. International Journal of Machine Tools and Manufacture, 47(14):2153-2161, 2007.

11. Berend Denkena, Thilo Grove, Marc-André Dittrich, D Niederwestberg, and M Lahres. Inverse determination of constitutive equations and cutting force modelling for complex tools using oxley's predictive machining theory. Procedia CIRP 31 (2015), 31:405-410, 2015.

12. Nikolaos E Karkalos and Angelos P Markopoulos. Determination of johnson-cook material model parameters by an optimization approach using the fireworks algorithm. Procedia Manufacturing, 22:107-113, 2018.

13. John E Plumeri, Lukasz Madej, and Wojciech Z Misiolek. Constitutive modeling and inverse analysis of the flow stress evolution during high temperature compression of a new ze20 magnesium alloy for extrusion applications. Materials Science and
Engineering: A, 740:174-181, 2019.

14. Hemanth K Amarchinta, Ramana V Grandhi, Allan H Clauer, Kristina Langer, and David S Stargel. Simulation of residual stress induced by a laser peening process through inverse optimization of material models. Journal of Materials Processing Technology, 210(14):1997-2006, 2010.

15. Ashkan Mahmoud Aghdami and Behnam Davoodi. An inverse analysis to identify the johnson-cook constitutive model parameters for cold wire drawing process. Mechanics \& Industry, 21(5):527, 2020.

16. Mathias Agmell, Aylin Ahadi, and Jan-Eric Ståhl. Identification of plasticity constants from orthogonal cutting and inverse analysis. Mechanics of $M a-$ terials, 77:43-51, 2014.

17. Jinqiang Ning, Vinh Nguyen, Yong Huang, Karl T Hartwig, and Steven Y Liang. Inverse determination of johnson-cook model constants of ultra-finegrained titanium based on chip formation model and iterative gradient search. The International Journal of Advanced Manufacturing Technology, 99(5-8):1131-1140, 2018.

18. Tuğrul Özel and Yiğit Karpat. Identification of constitutive material model parameters for highstrain rate metal cutting conditions using evolutionary computational algorithms. Materials and manufacturing processes, 22(5):659-667, 2007.

19. Rocco Eisseler, Konstantin Drewle, Karl Christoph Grötzinger, and Hans-Christian Möhring. Using an inverse cutting simulation-based method to determine the johnson-cook material constants of heattreated steel. Procedia CIRP, 77:26-29, 2018.

20. Aviral Shrot and Martin Bäker. A study of nonuniqueness during the inverse identification of material parameters. Procedia CIRP, 1:72-77, 2012.

21. Michael Storchak, Philipp Rupp, Hans-Christian Möhring, and Thomas Stehle. Determination of johnson-cook constitutive parameters for cutting simulations. Metals, 9(4):473, 2019.

22. SPFC Jaspers and JH Dautzenberg. Material behaviour in conditions similar to metal cutting: flow stress in the primary shear zone. Journal of Materials Processing Technology, 122(2-3):322-330, 2002.

23. Donald R Lesuer, GJ Kay, and MM LeBlanc. Modeling large-strain, high-rate deformation in metals. Technical report, Lawrence Livermore National Lab., CA (US), 2001.

24. DI Lalwani, NK Mehta, and PK Jain. Extension of oxley's predictive machining theory for johnson and cook flow stress model. Journal of Materials Processing Technology, 209(12-13):5305-5312, 2009.

25. Industrial Administration, Engineering Production Group Applied Mechanics Group, and 
G Boothroyd. Temperatures in orthogonal metal cutting. Proceedings of the Institution of Mechanical Engineers, 177(1):789-810, 1963.

26. Leon Lasdon, Abraham Duarte, Fred Glover, Manuel Laguna, and Rafael Martí. Adaptive memory programming for constrained global optimization. Computers $\mathcal{E}$ operations research, 37(8):15001509, 2010.

27. Russell Eberhart and James Kennedy. Particle swarm optimization. In Proceedings of the IEEE International Conference on Neural Networks, volume 4, pages 1942-1948. Citeseer, 1995. 